DOI: 10.35784/IAPGOS.235

\title{
APPLICATION OF CLONAL SELECTION ALGORITHM FOR PID CONTROLLER SYNTHESIS OF MIMO SYSTEMS IN OIL AND GAS INDUSTRY
}

\author{
Olga Shiryayeva, Timur Samigulin \\ Satbayev Kazakh National Research Technical University, Automation and Control Department, Almaty, Kazakhstan
}

Abstract. This paper presents the results of the Smart technologies application to the synthesis of MIMO-systems in oil and gas industry. In particular, there is considered a multidimensional multiply connected system for gas distillation process control through a distillation column with regulators configured on the basis of Smart-technologies - clonal selection algorithm (CLONALG) of an artificial immune system (AIS).

Keywords: artificial immune system (AIS), clonal selection algorithm (CLONALG), PID-controller, MIMO-system

\section{ZASTOSOWANIE ALGORTYMU SELEKCJI KLONALNEJ DO SYNTEZY REGULATORA PID SYSTEMÓW MIMO W PRZEMYŚLE PETROCHEMICZNYM}

Streszczenie. W artykule przedstawiono wyniki zastosowania inteligentnych technologii do syntezy systemów MIMO w przemyśle petrochemicznym. W szczególności rozważany jest wielowymiarowy układ sterowania procesem destylacji gazu w kolumnie destylacyjnej z regulatorami skonfigurowanymi na podstawie tzw. inteligentnych technologii-algorytmu doboru klonów (CLONALG) sztucznego uktadu immunolgicznego (AIS).

Słowa kluczowe: sztuczny układ immunologiczny (AIS), algorytm doboru klonów (CLONALG), kontroler PID, system MIMO

\section{Introduction}

The problem of synthesis of complex, that is, multidimensional, multi-connected, technological processes control is an urgent task in control theory. A promising direction in the design of MIMO-systems is the use of bioinspired algorithms that are effectively used in solving optimization problems [15].

Nowadays, a large amount of publications are devoted to the synthesis of optimal control based on bioinspired algorithms for one-dimensional (SISO) systems [2]. Genetic algorithms (GA) $[10,14]$, ant colony algorithms (ACO) [5], particle swarm algorithms (PSO) [1], artificial immune systems (AIS) [6] are widely used. The considered algorithms have the following distinctive features: high accuracy of calculations, speed, and the ability of the algorithms to work at the absence of complete information about the control object.

Of a particular interest are the algorithms for regulators tuning based on AIS. In particular, the CLONALG algorithm, the use of which for solving optimization problems and regulators tuning gives more efficient results than ACO and GA. In the article [12], the simulation results show that the algorithm of the immune system for tuning the PID-regulator has a high accuracy of the solution and the rate of convergence.

The use of an artificial immune system algorithm for optimization problems is presented in [4], as an example of which there was taken a DC servo drive speed control. The PID-regulator controller was configured using the AIS algorithm by minimizing the absolute time error. The results were compared with the results of the Ziegler-Nichols tuning method, and it was obvious that the AIS gave the best results. The AIS algorithm showed that it is able to find a global optimal solution, and it gave a better answer than the traditional tuning methods in terms of time rise, time adjustment, steady state error and overshoot.

The works $[11,16]$ are also devoted to the tuning of a PIDregulator of the systems using the AIS algorithm. The simulation results show that the AIS algorithm is an effective method for PID-regulator parameters optimization.

Among the AIS algorithms, the CLONALG algorithm is used extensively. Clonal selection is a theory that is used to explain the mechanisms of the immune response when immune cells recognize images of foreign antigens by antibodies [9]. The CLONALG algorithm, which models the basic principles of the clonal selection theory of the immune system, belongs to the category of computational intelligence systems, which include systems capable to adapt their behaviour when they achieve their goals, i.e. which have the ability to learn even at the absence of a person, in contrast to artificial intelligence systems and to so-called "symbolic computations", the latter of which are incapable of self-learning in general.

The CLONALG algorithm is of a great interest because of its development on a class of MIMO control systems. The purpose of this article is to obtain basic concepts for the formation of optimal control synthesis algorithms based on the CLONALG clonal collection algorithm of artificial immune systems for a MIMO control system.

\section{Mathematical model of MIMO-system in oil and gas industry}

As a MIMO control system, let consider a two-dimensional process of oil and gas industry, characterized by the presence of two inputs and two interconnected outputs.

For multidimensional multiply connected system for gas distillation process control through a distillation column, it is necessary to synthesize a controller based on bioinspired algorithms in order to achieve the desired modes as quickly as possible.

Distillation columns consist of several components, each of which is used to transfer thermal energy or to increase mass transfer [13]. Basic DC consists of: a vertical apparatus containing plates, which are designed to enhance the separation of components; a reboiler (boiler for reheating) to provide thermal energy in order to achieve the necessary evaporation in the lower part of the column; a condenser for cooling and condensing steam from the top of the column; a reflex tank (column irrigation collector) to hold the condensed steam, in order to return the fluid back from the top of the column. Most distillation process control systems, both conventional and modern, suggest that the column operates at constant pressure. The pressure fluctuation makes the control more flexible and ensure its efficiency. The structure of the $L-V$ ( $L$ is the flow rate of the liquid, $V$ is the flow rate of steam) can be considered as the standard control structure for distillation with double control over the content. In such a control structure, the flow rate of the liquid $L$ and the flow rate of s steam $V$ are control inputs [13]. The task of the regulator is to maintain the desired concentration of the products, $\chi_{B}, \chi_{D}$, at the output of the unit. 
Let's mathematic model of distillation process is described by equation [13]:

$$
\left|\begin{array}{l}
\chi_{D}(s) \\
\chi_{B}(s)
\end{array}\right|=\frac{1}{1+1.9588 s}\left|\begin{array}{cc||}
0.0042 & -0.0062 \\
-0.0052 & 0.0072
\end{array}\right| \begin{array}{|c}
L(s) \\
V(s)
\end{array} \mid
$$

where $L$ is the flow rate of the fluid, $V$ the flow rate of steam, $\chi_{B}$ the liquid concentration in bottom, $\chi_{D}$ the liquid concentration in the distillate.

In accordance with (1) let introduce the notation for two controlled inputs, $u_{1}, u_{2}$, and two output signals $y_{1}, y_{2}$ :

$$
\begin{aligned}
& \left|\begin{array}{l}
u_{1}(s) \\
u_{2}(s)
\end{array}\right|=\left|\begin{array}{l}
L(s) \\
V(s)
\end{array}\right|, \\
& \left|\begin{array}{l}
y_{1}(s) \\
y_{2}(s)
\end{array}\right|=\left|\begin{array}{l}
\chi_{D}(s) \\
\chi_{B}(s)
\end{array}\right|
\end{aligned}
$$

Let $G_{11}, G_{12}, G_{21}, G_{22}-$ plant transfer functions, connecting inputs and outputs:

$$
\left|\begin{array}{l}
y_{1} \\
y_{2}
\end{array}\right|=\left|\begin{array}{ll}
G_{11} & G_{12} \\
G_{21} & G_{22}
\end{array}\right| \cdot\left|\begin{array}{l}
u_{1} \\
u_{2}
\end{array}\right|=\left|\begin{array}{cc}
\frac{0.0042}{1.9588 s+1} & \frac{-0.0062}{1.9588 s+1} \\
\frac{-0.0052}{1.9588 s+1} & \frac{0.0072}{1.9588 s+1}
\end{array}\right| \cdot\left|\begin{array}{l}
u_{1} \\
u_{2}
\end{array}\right|
$$

Let consider a mathematical model of isolated subsystems:

$$
\left|\begin{array}{l}
y_{1} \\
y_{2}
\end{array}\right|=\left|\begin{array}{cc}
G_{11} & 0 \\
0 & G_{22}
\end{array}\right| \cdot\left|\begin{array}{l}
u_{1} \\
u_{2}
\end{array}\right|=\left|\begin{array}{cc}
\frac{0.0042}{1.9588 s+1} & 0 \\
0 & \frac{0.0072}{1.9588 s+1}
\end{array}\right| \cdot\left|\begin{array}{l}
u_{1} \\
u_{2}
\end{array}\right|
$$

For isolated subsystems (4), we synthesize optimal control based on the CLONALG algorithm.

\section{CLONALG for isolated subsystems of MIMO- system}

Problem statement. For isolated subsystems of the MIMOsystem (4), it is needed to synthesize the PID-controllers based on the CLONALG algorithm, which minimizes the integral quadratic quality criterions

$$
f_{i}=\int_{t=0}^{\infty} e_{i}^{2}(t) d t
$$

where $e(t)$ is a difference between the output and the reference signal, $i$ the subsystem number.

The CLONALG algorithm is often used to solve optimization and image recognition problems, reminding a parallel algorithm with climbing to the top and a genetic algorithm without a crossover operator [9]. The algorithm was developed on the basis of the immune system processes and its clonal-selective theory. In the context of this theory, the immune system produces antibodies in response to the intrusion of foreign antigens. Antibodies multiply in proportion to the degree of recognition: the better the antigen detection process is, the more antibodies the system generates (the cell reproduction process starts) [7].

During the reproduction process, individual cells undergo a mutation process, which allows them to have a higher affinity for a recognizable antigen: the higher the affinity of the parent cell is, the less they mutate, and vice versa. The main immune mechanisms in the development of the algorithm are the processing of a certain set of antibodies, the removal of antibodies with low affinity, maturation of affinity and the repeated selection of clones in proportion to their affinity for antigens [3].

Specifically it is the Euclidean distance between the vectors represented by an exemplar of the system and a training instance. In the case of a nominal value, the attribute distance is taken as a Boolean match (0) no match (1). Other attribute specific distance measures can be used such as temporal difference for date attributes and edit difference for string attributes or Hamming distance is bit strings are used.
The conceptual basis for the application of the CLONALG algorithm for solving the synthesis of typical regulators for MIMO-systems is the formation of concepts corresponding to the theory of immune systems and to the algorithm of clonal selection. In the context of the problem statement for the control system (4), the generalized form of antibodies corresponds to the vector of the arguments $A b=\left(e_{1}, e_{2}, u_{1}, u_{2}\right)$, and presents a set of solutions; as antigens there is used the quality criterion, presented as functions: $A g=f\left(e_{1}, e_{2}, u_{1}, u_{2}\right)$. For the synthesis of typical regulators for MIMO-systems, we use the quadratic integral quality criterion corresponding to the Lagrange task.

Formally, the clonal selection algorithm for solving the problems of optimal control synthesis of the complex systems can be represented as [14]:

$$
C L O N A L G=(A g, A b, G, S, C, M, f, d, t)
$$

Where $A g$ is a subset of antigens, $A b$ population of antibodies, $G$ representation of space, $S$ selection operator, $C$ cloning operator, $M$ mutation operator, $f$ quality criterion, $d$ amount of antibodies which should be replaced by the new ones, $t$ generation number.

In order to solve the problems of optimal control systems synthesis, this algorithm is used in the sequence shown on figure 1 [8].

At the initialization stage, there is randomly generated the initial set of solutions, controllers gains, that satisfy the criterion (5).

The next step is the selection of $n$ antibodies with better affinity, after which there is carried out the selection of antibodies suitable for cloning:

$$
N_{C}=\sum_{i=1}^{n} \operatorname{round}\left(\frac{\beta N}{i}\right)
$$

Where $\beta$ is multiplier that controls the amount of clones, $N$ the total amount of antibodies, $N_{C}$ the number of clones created by each selected antibody.

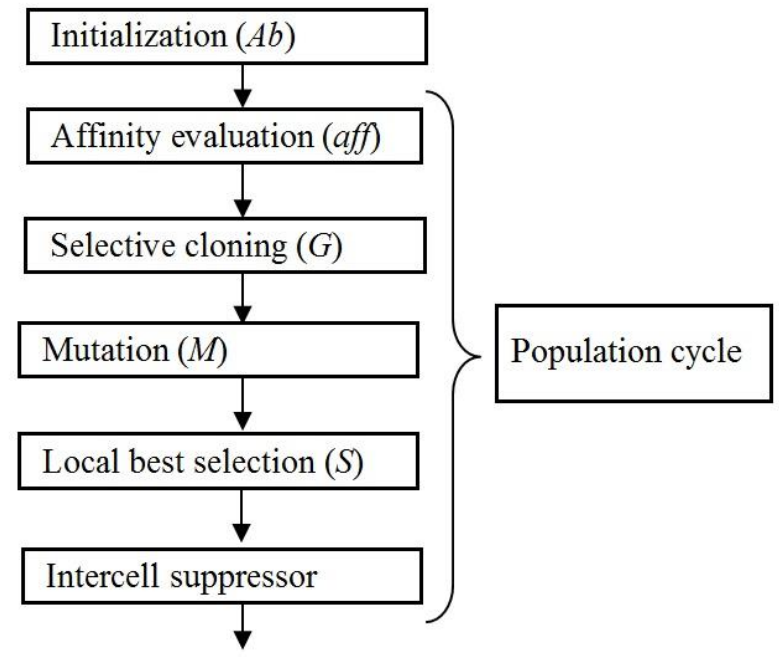

Fig. 1. The flowchart of CLONALG for control system

As a result of the cloning of antibodies with high affinity, we obtain a set that undergoes random mutation. If the degree of affinity is high, then the mutation rate is low:

$$
A b_{i, m}=A b_{i}+A \cdot(\operatorname{rand}[-1,1]) \cdot\left(A b_{\max }-A b_{\text {min }}\right)
$$

where $A$ is a factor dependent on affinity, which decreases with affinity increasing.

Low-affinity antibodies are replaced by the new, randomly generated antibodies, represented by the expression:

$$
A b_{i}=A b_{\text {min }}+(\operatorname{rand}[0,1]) \cdot\left(A b_{\text {max }}-A b_{\text {min }}\right)
$$

As a result of the CLONALG algorithm application there are many solutions with a high degree of affinity. These decisions are the controllers gains, $P_{i}, I_{i}$, of system (4): 


$$
u_{i}(t)=P_{i} e_{i}(t)+I_{i} \int_{t=0}^{\infty} e_{i}(t) d t \quad i=\overline{1,2}
$$

With transfer functions, $G_{C 11}, G_{C 22}$ :

$$
G_{C i i}(s)=P_{i}+I_{i} \frac{1}{s}, \quad i=\overline{1,2}
$$

where $P_{i}, I_{i}, i=\overline{1,2}$ are controllers' gains.

Using the MATLAB environment, the coefficients of the existing PI controllers (11) are calculated. For this, the following parameters are set for the CLONALG: $P_{1}=25 ; P_{2}=20$; $I_{1}=20 ; I_{2}=20$.

\section{Decoupling and modelling of MIMO-system in oil and gas industry with controllers}

In order to include synthesized controllers in the scheme of a MIMO-system with interconnections (3), there is applied the decoupling procedure. The main idea of this procedure is to use additional controllers to compensate the effect from interconnections, in order to reduce their interaction in the control loop. Ideally, decoupling control allows to affect only the desired variables.

Dynamically decoupled systems - a system in which each output depends on one and only one input. If all the cross-transfer functions are zero or negligible, the multi-loop system degenerates into a set of independent (decoupled) single-loop systems.

Let the matrix $H(s)$ will be the decoupling matrix with the parameters $H_{C 12}$ and $H_{C 21}$. In accordance with this, it is necessary to find the parameters of $H_{C 12}$ in order to eliminate the influence of the control interaction $u_{2}$ on the output $y_{1}$ :

$$
G_{p 11} H_{C 12} u_{22}+G_{p 12} u_{22}=0
$$

Similar to formula (12), the equations for the second input are:

$$
G_{p 22} H_{C 21} u_{11}+G_{p 21} u_{11}=0
$$

For the system (3) with controllers (11) the decoupling matrix consists of the following elements:

$$
H_{C 12}=-13 ; \quad H_{C 21}=-26
$$

The simulation scheme of the AIS based on the CLONALG for a MIMO-process in the MATLAB environment is shown on Figure 2. Decoupling-gain blocks are elements of a decoupling controllers, $H(s)$. At full decoupling, the multidimensional system is divided in such a way that there aren't interactions in control systems.

This stage of decoupling is the basis of the concept of CLONALG algorithms use for solving the problems of optimal control synthesis.

The simulation results of the MIMO-system with controllers and decoupling-gains are presented on Figure 3.

During the simulation of step responses, it was concluded that the use of decoupling control method allows to compensate the effect of relationships on the processes of a MIMO-system. Using decoupling operations for a MIMO-system allows efficient use of algorithms of Smart-technology.

The decoupling procedure is also based on optimization algorithms. In the future, it is intended to use the clonal selection algorithm of artificial immune systems to obtain decoupling coefficients.

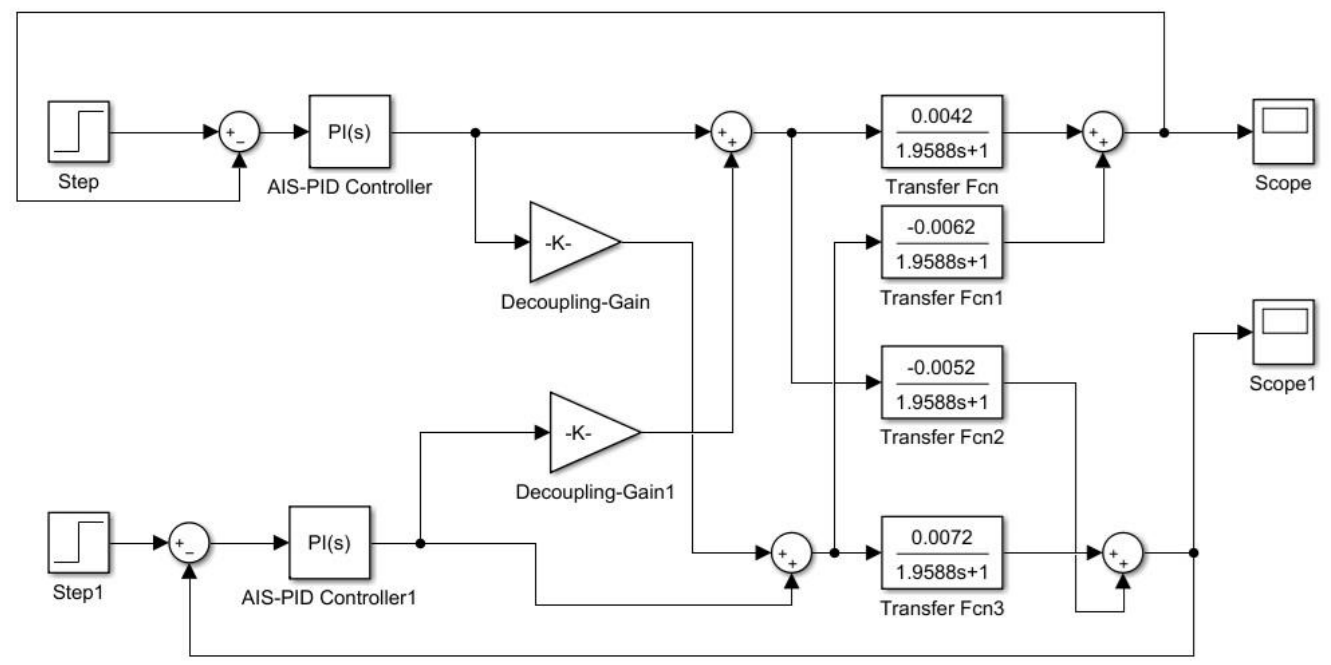

Fig. 2. The control system scheme in MATLAB environment
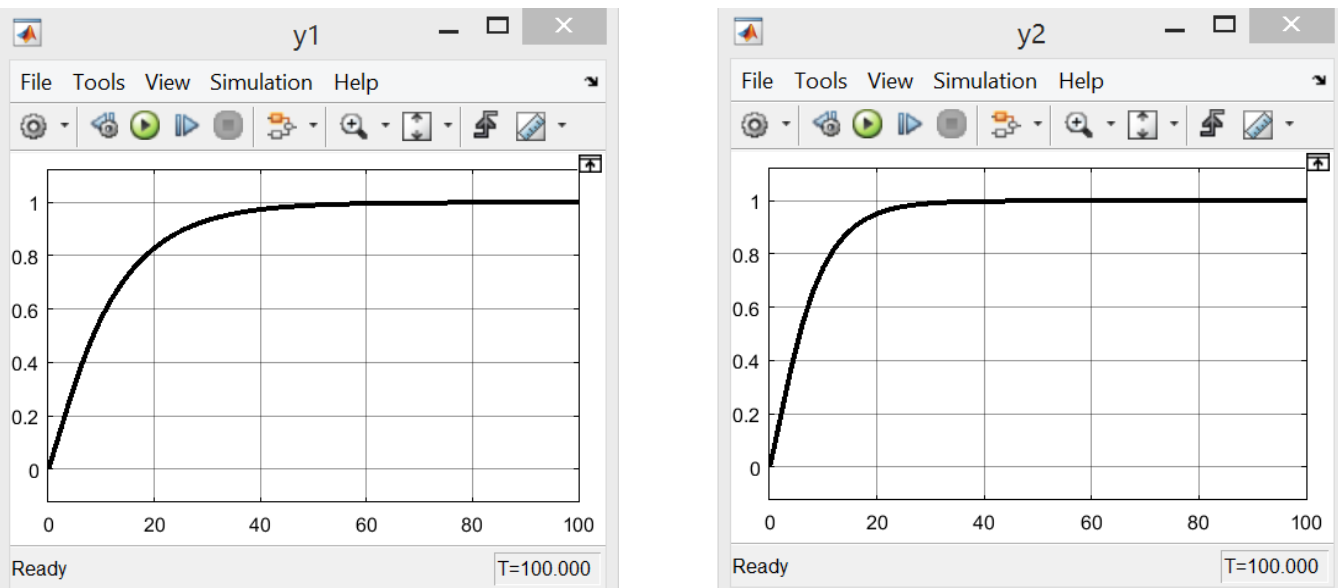


\section{Conclusion}

As a result of this work, there were obtained the results of the basic concepts formation of using the CLONALG algorithm for solving problems of optimal control of MIMO-systems in oil and gas industry. As a result of the CLONALG algorithm application there are gains of PID-controllers. In order to include synthesized controllers in the scheme of a MIMO-system with interconnections, there is applied the decoupling procedure.

In the future, it is planned to obtain the results of applying the algorithms of artificial immune systems in order to implement the procedure for MIMO-systems decoupling.

\section{Acknowledgements}

The work was carried out under the Grant of the Scientific Committee of the Ministry of Education and Science of the Republic of Kazakhstan on the subject: "Development of cognitive Smart-technology for intelligent MIMO-systems based on the approaches of artificial intelligence" No. AP05130018 (2018-2020).

\section{References}

[1] Arain B.A., Shaikh M.F., Harijan B.L., Memon T.D., Kalwar I.H.: Design of PID Controller Based on PSO Algorithm and Its FPGA Synthesization. International Journal of Engineering and Advanced Technology 2/2018, 201207.

[2] Bobikov A.I.: Correction of the weight matrices of the SURSD controller using bioinspired optimization algorithms. Bulletin of the Russian State Technical University 55/2016, 77-83.

[3] Burlakov M.E.: Overview of the basic algorithm of the artificial immune system on the theory of negative selection. Ufa: Collection of articles of the International Scientific and Practical Conference 2014, 29-31.

[4] Castro L., Zuben F.: The Clonal Selection Algorithm with Engineering Applications. Proceedings of Workshop GECCO'00, Las Vegas 2000, 36-37.

[5] Chinjiang L.: Optimal design of high-rise building wiring based on ant colony optimization. Cluster Computing 2018, 1-8.

[6] Dasgupta D., Yu S., Nino F.: Recent advances in artificial immune systems models and applications. Applied Soft Computing Journal 2/2011, 1574-1587.

[7] Kushnir N.V., Kushnir A.V., Anatskaya A.V., Katysheva P.A., Ustinov K.G. Artificial immune systems: an overview and state of the art. Scientific works of KubGTU 12/2015, 10

[8] Li Zh., Huang H., Tan H., Zhang Y.: IA-AIS: An Improved Adaptive Artificia Immune System and Its Application in Tuning of PID Controlled System. Journal of Information \& Computational Science 5/2008, 2193-2200.

[9] Litvinenko V.I., Fefelov A.A., Goravsky S.P.: Object-oriented implementation of the clonal selection algorithm. Radio electronics. Computer science. Management 1/2003, 81-88.
[10] Minian F, Sabouhi H., Hushmand J., Hallaj A., Khaledi H., Mohammadpour M.: Gas turbine preventive maintenance optimization using genetic algorithm. International Journal of System Assurance Engineering and Management $8 / 2016,594-601$

[11] Sahraoui M., Salem M.: Application of artificial immune algorithm-based optimisation in tuning a PID controller for nonlinear systems. International Journal of Automation and Control 3/2015, 186-200.

[12] Saleh M., Saad S.: Artificial Immune System based PID Tuning for DC Servo Speed Control. International Journal of Computer Applications 2/2016, 23-26.

[13] Shiryaeva O.I., Samigulin T.I.: Development of SMART-management system of a complex object of the oil and gas industry using the decoupling procedure. Bulletin of KazNRTU 5/2017, 50-55.

[14] Slavov T., Roeva O.: Application of Genetic Algorithm to Tuning a PID Controller for Glucose Concentration Control. WSEAS Transactions on Systems 7/2012, 223-233

[15] Trebuhin A.V.: Methods for solving optimization problems using bioinspired algorithms. Young Researcher Don, DSTU 6/2017, 108-111.

[16] Wang M., Feng S., He Ch., Li Zh., Yu X.: An Artificial Immune System Algorithm with Social Learning and Its Application in Industrial PID Controller Design. Mathematical Problems in Engineering 2017, 13.

\section{Ph.D. Olga Shiryayeva}

e-mail: o.shiryayeva@ satbayev.university

She received the Ph.D. degree in electrical engineering from the Satbayev University, Almaty, Kazakhstan, in 2001. Since 2002, she has been an Associate Professor with the Automation and Control Department, the Satbayev University, Almaty, Kazakhstan. Her current research interests include artificial immune systems, evolutionary algorithms, robust control systems, and MIMO-systems. In 2017 was awarded a "Better lecturer of Kazakhstan - 2017".

ORCID ID: 0000-0003-2532-0636

\section{M.Sc. Timur Samigulin}

e-mail: timur.samigulin@yandex.kz

He received the M.Sc. degree in electrical engineering from the Satbayev University, Almaty, Kazakhstan, in 2018. Now he is PhD student with the Automation and Control Department. His current research interests include smart control and forecasting systems, algorithms and software for smart systems. He was awarded a scholarship from Chevron company. In 2017 and in 2018 won scholarships of the first president of the Republic of Kazakhstan. In 2019 , studied at the Honeywell Automation School in Amsterdam.

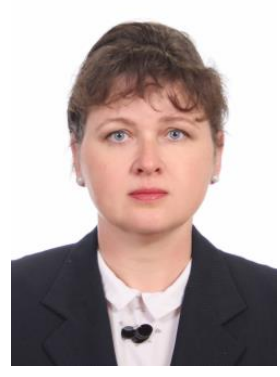

ORCID ID: 0000-0001-9963-6719

otrzymano/received: 30.05 .2019

przyjęto do druku/accepted: 15.06 .2019 